\title{
LA RECONSTRUCCIÓN DEL ESPEJO: 1969 ¿EL INDÍGENA COMO PERSONA?
}

\section{Luisa Fernanda García Lozano*}

\author{
Fecha de recibido: 31 de octubre de 2012 \\ Fecha de aprobado: 2 de abril de 2013 \\ Artículo de reflexión
}

Forma de citación: García, L. F. (2013). La reconstrucción del espejo: 1969. ¿El indígena como persona? Revista Prolegómenos. Derechos y Valores, 16, 31, 87-97.

\begin{abstract}
Resumen
Parte de las hipótesis que sustentan los desarrollos de la sociología jurídica en Colombia consisten en dar cuenta de la distancia del derecho y la sociedad, así como comprender la incidencia del derecho en las realidades sociales, por lo tanto, este se ha convertido en su objeto de estudio por excelencia. En este sentido, con el fin de establecer dicha relación, mediante la recopilación de fuentes periodísticas de la época de 1969 a 1970, se buscó reconstruir el imaginario social en cuanto a las ideas y pensamientos que en una determinada región y tiempo se tenían frente al atributo de la personalidad, específicamente, del indígena en Colombia. Para ello, a partir de un particular suceso llevado a la justicia penal, la Masacre de la Rubiera, se reconstruyó aquella fatídica percepción acerca de la personalidad del indígena. Como un fenómeno de reflejo producido por un espejo, esta herramienta metafórica de las Ciencias Sociales permite estudiar, tras este suceso histórico, a la institución encargada de cimentar control social: el Derecho. Institución que, socialmente hablando, es la encargada de describir y atribuir personalidad, como categoría jurídica, a los ciudadanos.
\end{abstract}

\section{Palabras clave}

Personalidad, Espejo, Indígena, Derecho, Masacre de La Rubiera.

Docente e Investigadora. Abogada de la Universidad Santo Tomás de Colombia. Máster Oficial de la Universidad del País Vasco y de la Università degli Studi di Milano: Master in Sociology of Law ofrecido por el Instituto Internacional de Sociología Jurídica de Oñati. Actualmente se encuentra trabajando en varias universidades entre las que se encuentran la Universidad Santo Tomas y la Universidad Militar Nueva Granada. Correo electrónico: trevannian@gmail.com. El presente trabajo hace parte de la investigación que complementa la tesis doctoral. 


\title{
THE RECONSTRUCTION OF THE MIRROR: 1969 THE NATIVE AS A PERSON?
}

\begin{abstract}
Part of the different hypotheses that support the developments of law sociology in Colombia revolve around measuring the distance between law and society and understanding the influence of law on social reality, becoming the study object par excellence. In order to establish said relation, the article sought to rebuild the social imaginary regarding the ideas and thoughts of a particular region and time by collecting news sources between the years 1969 - 1970, about the personality attribute of the Colombian natives. To do this, the article rebuilds a particular event brought up to local criminal justice, "La Rubiera" massacre, to observe that fateful perception about the personality of the indigenous people. As a reflection phenomenon produced by a mirror, this metaphorical tool of social sciences allows to study, through this historic event, the responsible institution for sustaining social control: Law. Institution that, socially speaking, is responsible for attributing and describing personality, as a legal category, to the citizens.
\end{abstract}

\section{Keywords}

Personality, Mirror, Indigenous people, Law, "La Rubiera" massacre.

\section{RECONSTRUÇÃO DO ESPELHO: 1969 O INDÍGENA COMO PESSOA?}

\begin{abstract}
Resumo
Algumas das hipóteses que sustentam o desenvolvimento da sociologia jurídica na Colômbia são para dar conta da distância do direito e da sociedade, assim também para entender o impacto da lei sobre as realidades sociais, portanto, o direito se tornou o objeto para estudar por excelência. A este respeito, a fim de estabelecer esta relação, através da recolha de fontes de jornais do período de 1969-1970, buscou-se reconstruir o imaginário social em termos das ideias e pensamentos em uma determinada região e época para conhecer as características da personalidade do indígena na Colômbia. Para fazer isso, a partir de um incidente em particular levado para justiça criminal, a massacre da Rubiera, foi reconstruída aquela fatídica percepção sobre a personalidade do nativo. Como um fenômeno do reflexo produzido por um espelho, esta ferramenta metafórica das Ciências Sociais permite estudar, após este evento histórico, a instituição encarregada de cimentar a lei de controle social: o direito. Instituição que, socialmente falando, é responsável por descrever e atribuir personalidade, como uma categoria jurídica, aos cidadãos.
\end{abstract}

\section{Palavras-chaves}

Personalidade, espelho, indígena, Direito, Massacre da Rubiera. 


\section{INTRODUCCIÓN}

El espejo, a manera de elemento metafórico representativo de las Ciencias Sociales, ha servido como herramienta para representar una imagen concreta de la sociedad: "Las sociedades son la imagen que tienen de sí mismas vista en los espejos que construyen para reproducir las identificaciones dominantes en un momento histórico dado"(Caldas, 2004, p. 21). Por lo tanto, si se realiza una ejemplificación de dicha metáfora, un espejo representa tanto los imaginarios como las percepciones sobre las cuales la sociedad esta cimentada. Solo estos imaginarios serían reflejos de luz, por ello, el cerebro social organiza estos reflejos en elementos que pueda reconocer, a esa figura es lo que llamamos sociedad.

Entre los elementos más concretos o palpables que las personas reconocen se encuentran las instituciones, las cuales se componen de elementos tales como: cultura, roles, mores, costumbres, normas sociales que organizan las actuaciones sociales e, históricamente, la institución que ejerce la función de controlar por excelencia: el Derecho.

En este sentido, la hipótesis que contiene la afirmación anterior: "el derecho como institución, por ende manifestación de la cultura y la estructura social", conlleva a reflexionar si realmente el Derecho representa las manifestaciones culturales, si puede trasformar la realidad y si la imagen en el espejo constituye en un reflejo real.

El presente escrito nace de una inquietud que ha estado presente a lo largo de la vida académica de la autora y hace parte del problema de investigación de la tesis doctoral: la relación derecho y sociedad. La inquietud se precisa en los hechos paradigmáticos que envuelve la historia de Colombia, los cuales evidencian la ausencia de un reflejo con contenidos políticos, económicos y culturales, entre otros. La persona, como categoría jurídica, le permite al hombre tener atributos, capacidad y le con- cede, desde la perspectiva jurídica, ser un miembro activo. Sin embargo, esta categoría ha sido una construcción subjetiva a lo largo de la historia, la cual atiende a los conceptos de estatus y clase en una sociedad que busca construir la fórmula de estado-nación. En este sentido, el presente caso, se constituyeen un estudio de caso paradigmático que demostró las posibilidades del derecho para la materialización de garantías de los indígenas, pese a las costumbres sociales.

En el presente escrito se desarrollará el concepto "persona" que se vivía en Colombia para los años de 1967 a 1972, a través de los reflejos de los diferentes campos sociales: el cultural, el social y el componente jurídico. Para ello, se basa en un estudio de caso, un suceso que bien puede categorizarse como hito: "la masacre de la Rubiera". De esta forma, se busca reflexionar sobre la composición de estos reflejos y se buscará comprender cómo inciden los reflejos en la toma de decisiones con componente jurídico que lleguen a trastocar la eficacia del derecho como institución.

\section{PRIMER REFLEJO: LA CRÓNICA}

El excepcional escritor colombiano Germán Castro Caycedo, en su primer libro, Colombia Amarga (1976), expuso un acontecimiento, escrito en forma de crónica-periodística, el cual produjo una trasformación en el concepto de persona en Colombia para la década de 1970, permitiendo las trasformaciones que se vivieron en la década de 1990 con respecto a los individuos y comunidades indígenas. Además, este interesante texto brinda elementos de suma importancia para descubrir el espejo que se construyó entre el período 1969-1973.

La Rubiera relata la masacre adelantada por un grupo de seis llaneros y dos llaneras que dieron muerte a dieciséis indígenas de la casta Cuiba en la zona este del país para el año de 1967. Los "vaqueros"; como se les llama en algunos de los periódicos de la época, 
argumentaron reiteradamente su inocencia a lo largo de las versiones presentadas en juicio, pretendiendo excusarse en el desconocimiento de la calidad de personas de los indígenas.

La mañana del 25 de diciembre de 1967, bajando por el río, se divisaron tres canoas ocupadas por 18 indígenas de la comunidad Cuiba provenientes de la frontera entre Colombia y Venezuela. Cuando los vio Aguirre, le dijo a Jiménez, "matemos a esos bichos aquí camarita"; Jiménez le respondió: "Aquí no, camarita, porque se pueden escapar algunos" (Castro, 1976) . A partir de ese momento la historia de sangre se empezó a tejer sin embargo, este plan se asemejó al de los niños cuando van a matar hormigas o cuando existe una plaga y hay que acabarla, a los bichos o animales no se les invita a cenar.

En horas de la tarde, Aguirre y Jiménez se acercaron a un hato en la "Rubiera", donde informaron al administrador Luis Enrique Morín: "Unos indios vienen a robarse la yuca y matar cerdos; hay que darles muerte. El plan lo gestaron entre Eudoro Gonzales, Celestino Rodríguez, Cupertino Sogamoso, Pedro Ramón Santana, Luis Ramón Garrido y Elio Torrealba" (Castro, 1976, p. 49).

En este escrito no es necesario describir los hechos con mayor detenimiento en sus brutales minucias; los indígenas fueron llevados a la hacienda con la promesa de darles comida y después de la cena los llaneros se dispusieron a dar muerte a los dieciséis indígenas; en medio de disparos, los llaneros con macetas (garrote grueso), pistolas, cuchillos y machetes dieron muerte a catorce de los dieciséis: Ramoncito (30 años), Luisito (20 años), Cirila (45 años), Luisa (40 años), Chain (19 años), Doris (30 años), Carmelina (20 años), Guáfaro (15 años), Bengua (14 años), Aruse (10 años), Julio (8 años) Aide (7 años), Alberto ( 3 años) $y$ un niño sin nombre que estaba siendo amamantado por Doris.

\section{SEGUNDO REFLEJO: PERSONAS O BICHOS}

La normatividad relevante que regía para la fecha en la cual fueron cometidos los hechos era la Ley 89 de 1890, en la cual el artículo primero consagraba:La legislación general de la República no regirá entre los salvajes que vayan reduciéndose a la vida civilizada por medio de Misiones. En consecuencia, el Gobierno, de acuerdo con la Autoridad eclesiástica, determinará la manera como esas incipientes sociedades deban ser gobernadas.

Al realizar un análisis del discurso, se hace necesario resaltar las expresiones: "salvajes" e "incipientes sociedades". Estas son claras expresiones del pensamiento criollo de la época, producto de la colonización española. Igualmente, se hace necesario establecer quiénes son salvajes. Así, el adjetivo "salvaje", de acuerdo con la Real Academia de la Lengua Española: "Se dice del animal que no es doméstico, y generalmente de los animales feroces" (Real Academia Española, 2001). En este sentido, bajo los parámetros de la interpretación gramatical, la ey se expidió para animales, no para personas.

Asimismo, de acuerdo al Código Civil colombiano (1873), se estableció taxativamente, en su Artículo 33: "La palabra persona, en su sentido general se aplica a la especie humana, sin distinción de sexo." (Congreso de la República de Colombia, 1873). Desde una aplicación de los métodos de interpretación básicos, cabe analizar cómo no se hace extensivo el concepto de persona a la categoría salvaje; pues el significado es taxativo, en un momento histórico en el cual predominaban los criterios positivistas exegéticos que no permitían mayor distanciamiento vía interpretación de la literalidad del texto de la Ley.

En este sentido, partiendo de un análisis lógico, gramatical y sistemático, es claro que la expresión que se usó en la Ley 89 de 1890, tenía la connotación de no entender a los in- 
dígenas como personas y, por tanto, no les reconocía derechos.

No obstante, cuando se sigue analizando el texto de la Ley 89 de 1890, el Artículo 40 advierte una modificación a la concepción expuesta en el Artículo 1:

Los indígenas son asimilados por la presente Ley a condición de los menores de edad, para el manejo de sus porciones en los resguardos, podrán vender con sujeción a las reglas prescritas por el derecho común para la venta de bienes raíces de los menores de veintiún años; debiendo en consecuencia solicitarse licencia judicial justificándose la necesidad o utilidad. Obtenido el permiso, la venta se hará en pública subasta conforme a las disposiciones del procedimiento judicial.Serán nulas y de ningún valor las ventas que se hicieren en contravención a lo dispuesto en este artículo, así como las hipotecas que afecten terrenos de resguardo, aun hecha la partición de éstos (Congreso de la República de Colombia, 1890).

El análisis del artículo en cuestión representa un tránsito en la concepción estatal del indígena de salvajes animales, a personas incapaces-relativos. En un somero estudio del texto, se evidencia cómo, pese ser considerados menores de 21 años, es decir, pese a no tener capacidad plena para adquirir derechos y obligaciones, existe cierta necesidad para que los indígenas puedan celebrar contratos civiles.

Si bien se consideraba a los indígenas como animales, estos eran, por disposición de la Ley 89 de 1890, poseedores de territorio y tenían la posibilidad de realizar actividades económicas. Igualmente, se explica este hecho en la doctrina católica que acompaño al país desde 1886 en su concepción legalista y constitucional, dado que los indígenas considerados incapaces relativos solo eran aquellos que habían realizado la transición para ser hijos de Dios, adquirían un alma al haberse bautizado. Así, el concepto de la Iglesia se trasformó para 1869 , no obstante, el de la ley no.

Igualmente, es necesario considerar algunas leyes y decretos que se expidieron antes de la fecha en que se promulgó dicha Ley, en los cuales se hace evidente que los indígenas estaban considerados dentro del grupo social colombiano y, por tanto, dentro de la interpretación histórica, sistemática y lógica no era posible considerarlos como animales.

Por ejemplo, dentro de los registros históricos se encuentra que en la independencia de Colombia los indígenas eran reconocidos como parte sustancial de la sociedad y podían ser dueños de sus resguardos con plena autonomía de sus territorios, reconocimiento de sus derechos y su sistema educativo; así, el Decreto 5 de 1820 , cuando reconoció los territorios indígenas, dispuso el respeto por sus miembros, con las connotaciones antes descritas; igualmente, en el artículo 13, taxativamente se dispuso la prohibición a los curas, jueces políticos y cualquier persona servirse de las comunidades sin una justa remuneración. También, en un sentido amplio, se prohibió la esclavitud de indígenas.

\section{TERCER REFLEJO: CULTURA: TRADICIONES Y COSTUMBRES}

Con el fin de ejemplificar el pensamiento del llanero acerca del indígena, me permitiré citar en extenso el reflejo de la sociedad. Para ello, el siguente extracto publicado en el diario El Tiempo, periódico clásico y de amplia circulación nacional: Cuando el indio quiere comer ganado, se reúne con los de su grupo y persigue unas cuantas reses, a carrera tendida sobre la pampa, las que va desjarretando con macetas y luego con la flecha o el cuchillo las termina, para comer un poco y el resto dejarlo a los gallinazos, o como dicen en el llano, 'para la chulamenta'. Por esa circunstancia, el llanero consideraba al indio como un animal dañino y le asimilaba al tigre, clasificación cuya mayor 
peligrosidad corresponde al primero, pues el ganado ha aprendido a defenderse del tigre, toda vez que el indio contaba con métodos que el ganado no había contrarrestado, haciéndole letalmente peligroso. Ante indicios de la presencia de indios en los hatos, se organizaban cacerías humanas en las que tomaban parte los dueños de las fincas vecinas, $y$ armados de revólveres, escopetas y principalmente carabinas bala $U$, llamadas flower, emprendían la expedición cinegética, que prolongan hasta cuando hombres mujeres y niños indígenas son atravesados por las balas de los cazadores (Atuesta, 1972).

Las explicaciones antropológicas que se le han dado a este fenómeno han sido principalmente de origen colonizador, es decir, se ha interpretado como una consecuencia directa de la pugna por el territorio entre indígenas y colonos (Gómez, 1987). En una etapa posterior a la independencia, la principal necesidad de los llaneros era la distribución del territorio $y$, por ende, el cambio de estatus en relación a la posesión de bienes, así, los paradigmas adaptativos con los cuales cada grupo adelanta sus relaciones sociales han impedido la coexistencia de los mismos. Igualmente, las doctrinas confesionales impuestas a lo largo del Siglo XIX y XX hacían alusión al concepto de indígena como un ser irracional, bárbaro y con falta de moral; estos dogmas eran enseñados a los colonos y se constituyeron en valores para amplias zonas.

Los aspectos culturales antes descritos, conllevaron a que durante el proceso de la Rubiera, en la indagatoria, los sindicados manifestaran: "Desde pequeño a mí me enseñaron que los indios son dañinos y que hacen males. A mí me enseñaron a odiarlos. Hoy por medio de la civilización uno sabe que son cristianos igual a uno, yo no sospechaba eso antes, (entrevista al sindicado Luis Morín) (Castro, 1976, p. 46).

O, por ejemplo, que el Juez se sorprendiera y preguntase: ¿No cree que matar indios es malo?
Reo: yo no creí que fuera malo ya que son indios. Los indios de allá claro que no son tan belicosos, a la gente no le hacen nada pero si matan animales (...)

Juez: ¿Ha matado indios antes?

Reo: He matado a seis indios en el año de 1960 y los enterré en un sitio llamado el "Garcero"(...)

Juez: ¿Por qué lo hizo?

Reo: yo no sabía que eso era malo, que lo castigaban a uno, pues en caso contrario no lo hubiera hecho (Castro, 1976, p. 54).

Gran parte de la defensa se sostuvo en demostrar que se trataba de una práctica llanera, motivo por el cual se podría estructurar la inocencia de los implicados, por dicha razón, durante el proceso se hizo énfasis en las tradiciones del llano que se han venido explicando, e incluso, llegaron a declarar en juicio algunos íconos culturales de la época, como el compositor Miguel Angel Martin, entre otros.

\section{CUARTO REFLEJO: EL JUICIO}

Este proceso duró cerca de seis años en ser fallado definitivamente. Se compuso de dos juicios. El primero, declarado nulo, se realizó en Villavicencio; $y$, el segundo, se efectuó en la ciudad de Ibagué. Hay que tener en cuenta cómo, para ese entonces, en el país existían jurados de conciencia. Con el fin de aproximarse al desarrollo de los juicios, se realizó una búsqueda en los principales periódicos del país, principalmente El Tiempo, con el fin de estructurar la figura que reflejó el caso.

\subsection{Primer juicio (1972)}

El primer reporte que se realizó de forma oficial en el proceso de la Rubiera se publicó en enero de 1968, cuando un periódico nacional informó que en Venezuela se reportaba la muerte de Catorce Cuiberos (El Tiempo, 3 de enero de 1968). Paralelamente, El Espectador, otro diario de amplia circulación, informó acerca del "genocidio de 16 indios en el llano" (El 
Espectador, 3 de enero de 1968). Trascurrió un tiempo; y, hasta 1972, se dio inicio al proceso. Nuevamente se encontraron los artículos de prensa, la importancia de este juicio consistía en que, por vez primera, serían llevados a juicio personas por matar indígenas.

En las instalaciones donde se realizó el proceso hicieron presencia los jefes de varias comunidades indígenas, específicamente veinticuatro jefes indígenas (Franco, 1972). Entre ellos, se encontraban los gobernadores de Sibundoy, el gobernador de los Guambianos, de las comunidades de Cañamomo y Loma Prieta y los Arhuacos, su principal pretensión buscaba no dejar en la impunidad el caso. Sin embargo, el castigo, bajo su cosmovisión, debía ser otro: trabajo hacia la comunidad y exilio: "Trabajo para que no se queden en la cárcel comiendo y engordando; $y$, el exilio para que no regresen a Colombia a matar" (Castro, 1972).

El juicio se adelantó contra ocho de los once inculpados, los tres que huyeron fueron enjuiciados como reos ausentes. El argumento central de la defensa consistió en demostrar como la caza de los indígenas era una práctica cultural en la región y, por ende, los acusados no eran conscientes de que estaban cometiendo un delito; para ello, la defensa presentó expertos sociólogos, antropólogos e historiadores que confirmaban su hipótesis.

Tres hombres tenían la obligación de fallar, Armando Acosta, Ricardo Rueda y Oscar Rojas, quienes en medio de las opiniones y la presión social fallaron: "Inocentes por ignorancia invencible (...)"(Castro, 1972). Estos tres jurados, fuera de juicio, argumentaron: i) Armando Acosta, electricista de 42 años, fue cauchero en la zona del Vaupés y trabajó con indígenas: "Yo estoy de acuerdo con la defensa, la culpa la tiene el Estado y la iglesia por el abandono y el atraso en territorios nacionales", lo que más influyó en la decisión fue: "El análisis técnico, sociológico y sicológico de los grandes tratadistas de la criminología que hizo el defensor. Esto me llevó a darle en parte la razón". ii) Oscar Rojas, 41 años, inspector de tránsito nacido en Villavicencio:

En Colombia ha habido blancos que matan blancos en condiciones peores y algunas veces salen libres. No se puede pensar que por el hecho que resulta una persona absuelta, signifique que está autorizada para volver a matar (...) esto ha sido una lección suficiente para ellos y que es posible que ellos mismos en adelante van a divulgar que matar indios no es como matar animales (...) (Castro, 1972).

iii) Ricardo Rueda, 33 años, tipógrafo. Fue llanero pero ahora su vida trascurre en Villavicencio:

(...) lo que más influyó en mí, fue la ignorancia de los llaneros acusados, en el atraso del llano, y pensar en un Estado que se acuerda del hombre para aplícale la ley pero no para enseñarla (...)". Al preguntarle qué aprendió, expresó: “(...) que los indios en realidad es rencoroso y desagradecido (...) Es bueno matar indios? Malo es matar pero hay que saber en la situación en la que se mata" (Castro, 1972).

El fallo de primera instancia fue ampliamente cuestionado, la declaración de inocencia implicaba que el Estado no había trasformado sus prácticas de discriminación y exclusión. Por ello, varios actores sociales hicieron comunicados al respecto rechazando el caso, entre los más representativos se encuentran 450 profesores de distintas universidades y colegios del país y el Instituto Colombiano de Antropología e Historia (El Tiempo, 5 de Julio de 1972), quienes argumentaron que con fallos como estos se fortalecía la impunidad en el país.

Muchas versiones se discutían para poder encontrar la nulidad de un juicio, entre otro argumento se encontró que uno de los jurados de conciencia era inspector de tránsito, Oscar Rojas, y de acuerdo con "el artículo 528 de procedimiento penal, no pueden ser jurados de conciencia los funcionarios de policía judicial. Y el artículo 288 del mismo código, espe- 
cifica que quienes forman parte de la policía judicial, y allí están incluidos los inspectores de tránsito" (H.G.B, 3 de Julio de 1972).

Igualmente, el fiscal de los juzgados de Circuito Especializado de Bogotá, Jaime Vargas Moreno, denunció prevaricato por parte de uno de los jurados:

(...) el denunciado es Armando Acosta y el fiscal basa su denuncia en la publicación del tiempo en donde los antropólogos Víctor Daniel Bonilla y Gonzalo Castillo Cárdenas hacen graves cargos contra el jurado Acosta, consistentes en "las manifestaciones públicas que hizo desde el primer día de la audiencia que indicaban que ya en su ánimo estaba formado el veredicto absolutorio" (Morales, 1972).

En medio de las protestas y el afán por no dejar en firme la sentencia, se iniciaron algunas investigaciones del caso, el 2 de julio se nombró un investigador, quien señaló que el Juez:

Viola flagantemente el código de procedimiento penal por dos razones: primera, porque se resolvió o decidió la demanda por medio de un auto de substanciación, cuando debió hacerlo por interlocutorio y por no aceptar una petición del ordinal 6 artículo 27 del código penal (...) no se produjo la prueba pericial para declarar a los acusados inimputables, por estar por fuera de la civilización (El Tiempo, 2 de Julio de 1972).

En virtud de los resultados de las investigaciones y los hechos que se presentaron, el Juez Damaso Marenco Castillo declaró nulo el juicio, este hecho se reportó de la siguiente manera: "El juez dicto un veredicto de acuerdo con las atribuciones que le concede la ley dos días antes de que venciera el plazo con que contaba para hacer su pronunciamiento al respecto, el juicio se declaró contraevidente" (Morales, 1972).
Estas investigaciones al interior del proceso que tienen argumentos de corte procesal, en ningún momento se preguntaron por la persona del indígena y la necesidad de protegerlos. Cabe preguntarse: ¿en la región eran considerados personas? Finalmente el proceso fue declarado nulo y en virtud de la necesidad de buscar neutralidad en el proceso, este fue enviado para la ciudad de Ibagué.

\subsection{Segundo juicio - Fallo de noviembre de 1973}

Este segundo proceso no tuvo tanto cubrimiento periodístico, es muy poca la información que se encuentra de esta parte, al igual los datos que se suministraron fueron superficiales. En este orden de ideas, se conoce que el jurado fue conformado por un fotógrafo, un comerciante y un antiguo empleado del sector judicial, quienes declararon culpables a 7 de los 8 acusados, el Juez condenó a las penas máximas imponibles.

Dentro de los argumentos para realizar la defensa, se encuentran:

Este no se trata de un simple caso de página roja. Este proceso tiene dimensión nacional y aún continental, porque en afloran todas las manifestaciones de la problemática colombiana de las naciones subdesarrolladas (...) Se ha puesto aquí de presente que no la culpabilidad de los procesados, que son unas pobres víctima, el fruto de un medio ambiente, el fruto de un sistema político- económico inadecuado, la culpabilidad es colectiva: la de los dirigentes, la de quienes lanzan al pueblo por donde quiere marcha, la de quienes han labrado la desgracia masiva (Castro, 1973).

Asimismo, expresó frente al veredicto "el veredicto me sorprendí, pero no era raro, había mucha presión del gobierno, de la iglesia de la prensa. Querían que los comentaran para que la sociedad se lave las manos por todos los 
crímenes de indígenas durante más de cuatro siglos" (Ibíd.). Los acusados quedaron en silencio y solo Ramón Garrido, exclamó:

Todo lo bueno que han hecho con nosotros en seis años, lo destruyeron hoy. En ese tiempo nos habíamos civilizado, nos habíamos compuesto. Ganamos una experiencia, pero ahora solo nos queda el camino de la corrupción podrido en una cárcel. Saldremos viejos y desilusionados (...) (Castro, 1973).

De esta forma, terminó el caso que sería decisivo para el cambio de una concepción social frente a una comunidad específica y abrió las puertas para una serie de sucesos que posibilitaron el cambio jurídico-político que se evidencia en la Constitución Política de 1991.

\section{A MODO DE CONCLUSIÓN}

Paradójicamente, en el caso bajo estudio, todos los reflejos fueron concretamente señalados, descritos, y analizados. No obstante, la figura que se representa de la persona en la época está distorsionada, no se representa a un indígena como persona, el reflejo es una especie de centauro distorsionado, que busca, por medio de este juicio, adquirir un reflejo de lo que realmente es.

Los dos reflejos que chocan en la elaboración del concepto se encuentran entre el derecho y la cultura. De acuerdo a la investigación, estos elementos ,que cuando se combinaron chocaron, se repelieron y disputaron su permanencia en el contexto, las tradiciones coloniales y sus cosmovisión, no han sido elementos fáciles de superar a lo largo de la historia del país; por ende, las prácticas de discriminación y dominación racial han sido una constante en la historia hasta nuestros días.

La Ley 89 de 1890, describía, en su momento, las manifestaciones de poder y los valores que les eran implementados en la época. Los in- dígenas no eran considerados personas. Sin embargo, se hace evidente a través de la investigación como las trasformaciones sociales comienzan a permear las estructuras jurídicas y, por ende, con el trascurso del tiempo esta Ley perdió efecto al momento de fallar el caso emblemático, los dogmas impuestos habían cambiado, la modernidad para un país como Colombia traía consigo trasformaciones que no eran evidentes a los ojos de los mecanismos de poder, incluso al de la cultura los discursos de inclusión e igualdad estaban tomando fuerza en la región, las asociaciones de indígenas se estaban creando, lo cual producía un impacto político en las estructuras jurídicas.

Así, la ausencia del Estado en la otra Colombia era evidente, la desprotección hacia las comunidades, la ignorancia, así como la falta de desarrollo se reflejan en este caso, demostrando así que los procesos de desarrollo se vivían en diferentes momentos $y$, por tanto, se construían y reconstruían varios espejos en el mismo espacio tiempo. Por ejemplo, para la época se adelantaba uno de los mayores movimientos sociales que tenía el país, el cual produjo diversas modificaciones sociales, propugnando por la garantía de los derechos fundamentales. El movimiento indígena, cuya consolidación se da en las décadas de los 60 y 80 (Fajardo \& García, 2010).

La divergencia que se produce en los reflejos es lo que ocasiona la verdadera dislocación de la figura entre persona y animal. Es interesante, en el caso, establecer cómo, en el primer juicio, los jurados de conciencia que pertenecían a la misma región establecieron la inocencia de los victimarios en razón a su ignorancia, a las costumbres, al igual que la normatividad y usos de la región, matar indígenas no generaba castigo. En el segundo juicio, en razón de múltiples factores, tales como presión del gobierno, actores sociales y estar en otra región en donde el movimiento indígena hacia más presencia, como el Tolima, la decisión judicial se trasformó de tal manera que parecieron dos juicios diferentes. 
En este sentido, jurídicamente se postulan varios interrogantes acerca de la interpretación jurídica, la seguridad jurídica y el procedimiento; lo anterior, en virtud de que el reflejo jurídico se torna confuso cuando: en el primer juicio absuelven a los campesinos con un argumento cultural, bajo la teoría y concepto de Estado del momento, Estado de Derecho, por lo cual el argumento para cualquier acción jurídica dependía del mismo Derecho; asimismo, la declaración de nulidad del juicio sin elementos fácticos y el cambio de ciudad para realizar nuevamente el juicio afectan la seguridad jurídica, al igual que el procedimiento.

Estas ambivalencias en la estructura jurídica se convirtieron en una posibilidad para la modernidad, dado que, traspasa los enclaves culturales y jurídicos de la época abriendo paso a los discursos incluyentes que se fortalecen en las décadas posteriores.

Aunque este fallo fue uno de los más importantes para iniciar la trasformación que se vivió en la década de los noventa, la realidad en la región de los llanos no cambió mucho. En esta región olvidada se encuentran una gran cantidad de pueblos en peligro de extinción, entre otros: los Beyote, los Guayabero, los Hitnu y, por supuesto, los Cuibas que, a la fecha, estan compuestos por tan solo por 769 personas (ACNUR, 2011), quienes son víctimas de persecución y desplazamiento. Así, se evidencia uno de los principales problemas entre el derecho, la sociedad y la realidad, puesto que, las dinámicas que cada campo tienen, los lentos procesos de transformación, además de la falta de diálogo entre los campos sociales, produce en algunos casos que el derecho sea ineficaz o que las demandas culturales traspasen las normas nacionales e incluso internacionales.

Sin embargo, el caso en cuestión es de especial relevancia para estudiar la sociología jurídica, dado que, aun cuando en algunas ocasiones la cultura y el derecho distorsionan la imagen de la sociedad, al encontrarse, es posible verificar que las transformaciones jurídico políticas eficaces se retroalimentan de este tipo de hechos en donde el efecto jurídico y social de una sentencia produjo en el tiempo una transformación cultural.

\section{REFERENCIAS}

ACNUR (Agencia de las Naciones Unidas para los Refugiados). (2011). Informe sobre la situación de los pueblos indígenas en peligro de extinción. Extraído de http://www.acnur.org/ t3/fileadmin/scripts/doc.php?file=t3/fileadmin/Documentos/Pueblos_indigenas/2011/ Comunidades_indigenas_en_Colombia_-_ ACNUR_2011 en Agosto 22 de 2012.

Atuesta, H. (1972, Junio 13). Cacería del hombre por el hombre. El Tiempo, p.7.

Caicedo, G. (1972, Junio 09). Comenzó proceso de la rubiera. El Tiempo, p.15 A.

Caldas, A. (2004). La regulación jurídica del conocimiento tradicional la conquista de los saberes. Bogotá: Instituto Latinoamericano de Servicios Legales Alternativos.

Castro, G. (1972, Junio 22). Nuevo Receso en el juicio de la Rubiera. El Tiempo, 1 y 7A. Castro, G. (1972, Julio 2). Al juez le han tocado procesos sensacionales. El Tiempo.

Castro, G. (1972, Junio 29). Hablan los jurados. El Tiempo, p. 1 y $7 \mathrm{~B}$.

Castro, G. (1972, Junio 8). Los Acusados no duermen. El Tiempo, 9.

Castro, G. (1972, Julio 2). Continuan protestas por el juicio de la "Rubiera". El Tiempo, p. última $B$.

Castro, G. (1972, Junio 02). Investigador para el caso de la Rubiera. El Tiempo, p. última B. Castro, G. (1972, Junio 11). El proceso de la Rubiera: jefes indios piden castigo a colonos. El Tiempo, p.1 y 7A.

Castro, G. (1972, Junio 29). El juicio de la rubiera absueltos 7 acusados. El Tiempo, 1. 
Castro, G. (1973, Noviembre 7). Brillante defensa. El Tiempo.

Castro, G. (1973, Noviembre 7). El crimen de la Rubiera, seis condenados. El Tiempo.

Castro, G. (1973, Noviembre 7). Los procesados de la rubiera: nos destruyeron. El Tiempo. Castro, G. (1976). Colombia Amarga. Colombia: Planeta.

Congreso de la República de Colombia. Ley 89 de 1890. Por la cual se determina la manera como deben ser gobernados los salvajes que vayan reduciéndose a la vida civilizada. Noviembre 28 de 1890.

Congreso de la República de Colombia. Ley 57 de 1887. Por la cual se expide el Código Civil. s.f.

El Espectador. (1968, Enero 4). Envían comisiones al sitio donde se cometió el presunto genocidio en Arauca. El Espectador.

El Espectador. (1968, Enero 10). Venezuela y Colombia investigan asesinato de 17 indígenas. El Espectador.

El Espectador. (1968, Enero 3). Sin confirmar el genocidio de 16 indios en Arauca. El Espectador.

El Espectador. (1972, Junio 13). Plantean nulidad en el proceso por la matanza de 16 indios cuibas. El Espectador.

El Espectador. (1972, Junio 8). Hoy juicio a 14 Cuibas, asisten 24 jefes indígenas y el gobernador del Meta. El Espectador.

El Tiempo. (1968, Enero 3) 16 indios venezolanos muertos en colombia, las autoridades no tienen ninguna noticia. El Tiempo. p. 1 y 3.

El Tiempo. (1972, Julio 3). Obispado censura fallo sobre matanza de indígenas. El Tiempo.
El Tiempo. (1972, Julio 5). El juicio de la Rubiera: revocar fallo piden los profesores. El Tiempo.

El Tiempo. (1972, Julio 5). Hoy dictan contraevidencia. El Tiempo.

El Tiempo. (1972, Junio 10). Comenzó juicio por la Matanza de 16 Cuibas. El Tiempo, 11C.

El Tiempo. (1973, Noviembre 1). Juicio de la Rubiera: El martes, veredicto. El Tiempo.

Fajardo, L. \& García, L. (2010). Utopía y jurisprudencia constitucional. Un enfoque progresista de la Corte Constitucional Colombiana (1991-2000). Bogotá D.C.: Universidad Santo Tomás.

Franco, M. (1972, Junio 13). Defensor Anuncia una Carta fija. El Espectador.

Gómez, G. (1987). Llanos Oorientales: colonización y conflictos interétnicos. 18701970. Trabajo de tesis para optar por el título de Maestría en Historia Andina. Ecuador: Flacso.

H.G.B. (1972, junio 3). Vida judicial, el lío de la Rubiera. El Tiempo.

Morales, G. (1972, Junio 04). Denuncia por prevaricato contra jurado de la Rubiera. El Tiempo.

Morales, G. (1972, Junio 15). Veredicto sobre la Rubiera en dos días. El Tiempo, $11 \mathrm{~A}$.

Morales, G. (1972, Junio 16). Cuántos fueron los muertos? El Tiempo, 9.

Real Academia Española. (2012). Diccionarios de la lengua española. 22. Extraído de www.rae.es en agosto 23 de 2012.

Sánchez, A. (1973, Noviembre 01). Juicio de la Rubiera: Nulo, fallo del jurado. 